\title{
Hepatodiaphragmatic interposition of the colon
}

\author{
Huan-Lun Hsu MD, Kao-Lang Liu MD
}

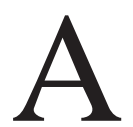

61-year-old woman presented with intermittent abdominal discomfort, which had been present for several weeks. She reported sometimes feeling better in the supine position than in the erect position. She had no relevant medical or family history and she took no medications. Physical examination showed a distended abdomen without tenderness, and reduced bowel sounds. The laboratory findings were unremarkable. A chest radiograph showed elevation of the right diaphragm and interposition of the ascending colon between the diaphragm and the liver (Figure 1). Contrast-enhanced computed tomography showed the hepatodiaphragmatic interposition of the ascending colon, with diffuse dilatation of bowel loops with no obstructive lesion seen (Appendix 1, available at www.cmaj.ca/cgi/content/full/cmaj $.092117 / \mathrm{DC} 1)$. Chilaiditi syndrome was diagnosed. The patient's symptoms improved with conservative treatment.

First described in $1910,{ }^{1}$ Chilaiditi sign is the asymptomatic interposition of the intestine, usually a portion of the hepatic flexure of the colon, between the liver and diaphragm, whereas Chilaiditi syndrome refers to clinical symptoms such as abdominal bloating, pain or anorexia developing from this phenomenon. ${ }^{2}$ Chilaiditi sign is generally considered an incidental radiographic finding, and its prevalence rate is reported to range from $0.025 \%$ to $0.28 \%$ and increased with age in one study. ${ }^{2}$ The postulated mechanism of this condition is an increase in space between the liver and the diaphragm. ${ }^{3}$ The interposition may appear only when the patient is in the erect position and resolve in the supine position. Several contributing factors may result in this congenital or acquired condition, including those related to the liver (congenital hypoplasia or reduction of liver volume due to cirrhosis), intestine (malrotation, adhesion or abnormal motility) and diaphragm (laxity of suspensory ligaments or nerve paralysis). Chilaiditi sign is also associated with older age (as in our patient), chronic lung disease, multiple pregnancies and acute weight loss. ${ }^{3}$ Whereas the interposition may occur temporarily without recurrence as a result of transient distention of the gut, most patients with Chilaiditi sign have persistent interposition, although only when in the erect position.

From the Department of Internal Medicine (Hsu), China Medical University Hospital, Taipei Branch, and the Department of Medical Imaging (Liu), National Taiwan University Hospital, National Taiwan University, College of Medicine, Taipei, Taiwan

CMAJ 2011. DOI:10.1503/cmaj.092117

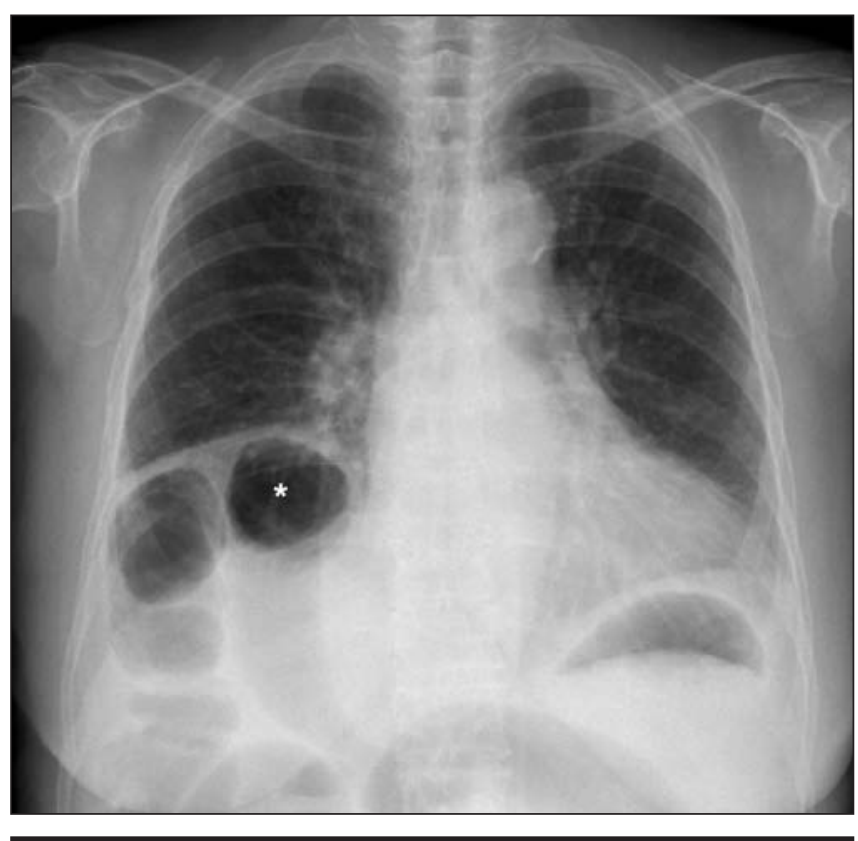

Figure 1: Standing chest radiograph of a 61-year-old woman showing elevation of the right diaphragm and interposition of the colon $(*)$ between the right diaphragm and the liver.

Conservative management for the syndrome includes bed rest and bowel decompression with rectal or nasogastric tube; surgical treatment may be required in cases of persistent pain, refractory ileus, colonic volvulus or bowel ischemia. Identification of the haustral folds or plicae circulares between the liver and diaphragm is crucial to avoid a misdiagnosis of pneumoperitoneum. ${ }^{4}$

This article has been peer reviewed.

Competing interests: None declared.

\section{REFERENCES}

1. Chilaiditi D. Zur frage der hapatoptose und ptose in allemeinen im auschluss an drei falle von temporaerer partiellaer leberverlagerung [article in German] Fortschr Gebiete Roentgenstrahlen 1910;11:173-208.

2. Orangio GR, Fazio VW, Winkelman E, et al. The Chilaiditi syndrome and associated volvulus of the transverse colon: an indication for surgical therapy. Dis Colon Rectum 1986;29:653-6.

3. Aldoss IT, Abuzetun JY, Nusair M, et al. Chilaiditi syndrome complicated by cecal perforation. South Med J 2009;102:841-3.

4. Alva S, Shetty-Alva N, Longo WE. Image of the month. Chilaiditi sign or syndrome. Arch Surg 2008;143:93-4. 